\title{
Análise da qualidade de uma rede altimétrica local
}

\author{
Quality analysis of a local network altimetry
}

\author{
Luciene Ferreira Gama ${ }^{1}$ \\ Ermerson de Vasconcelos Silva ${ }^{2}$ \\ Andréa de Seixas ${ }^{2}$ \\ ${ }^{1}$ Instituto Federal de Educação, Ciência e Tecnologia da Paraíba - IFPB , Unidade Acadêmica de Design, Infraestrutura \\ e Meio Ambiente, Curso Superior de Tecnologia em Geoprocessamento - IFPB Av. $1^{\circ}$ de maio, $n^{\circ} 720$, Jaguaribe, João \\ Pessoa-PB, 58015-430 \\ luciene.gama@ifpb.edu.br
}

${ }^{2}$ Universidade Federal de Pernambuco - UFPE, Centro de Tecnologia e Geociências - CTG, Departamento de Engenharia Cartográfica, Recife - PE Programa de Pós-Graduação em Ciências Geodésicas e Tecnologias da Geoinformação, Av. Acadêmico Hélio Ramos, s/n, Cidade Universitária, Recife-PE, 50740-530

ermerson.geoif@gmail.com, aseixas@ufpe.br

Recebido em 06/07/2015 - Aceito em28/06/2016

Received on 06/07/2015 - Accepted on 28/06/2016

RESUMO - Este trabalho tem como objetivo avaliar a qualidade de uma rede geodésica altimétrica de alcance local, para apoio as atividades de engenharia, utilizando nível analógico de precisão $\pm 2 \mathrm{~mm} / \mathrm{km}$ de duplo nivelamento e nível digital de precisão $\pm 0,3 \mathrm{~mm} / \mathrm{km}$ de duplo nivelamento, a partir das análises das Matrizes Covariâncias dos resíduos, das observações ajustadas e das altitudes ajustadas. Comparando-se em seguida as altitudes e desníveis ajustados e determinadas com o nível analógico com as altitudes e desníveis ajustados e determinados com o nível digital. Ressaltase que esta rede altimétrica não integra o Sistema Geodésico Brasileiro (SGB), sendo composta por doze Referências de Nível (RRNN), implantadas e localizadas no Centro Histórico da cidade de João Pessoa - PB. As Referências de Nível foram determinadas pelo método do nivelamento geométrico e ajustadas pelo Método dos Mínimos Quadrados, utilizando o modelo dos correlatos. Em relação às incertezas obtidas para as coordenadas altimétricas dos pontos de referência implantados através do método de nivelamento geométrico com o nível analógico, estas são melhores que $\pm 0,001 \mathrm{~m}$. Desta forma, a rede altimétrica implantada com o nível analógico (precisão alta) e comparada com a rede altimétrica implantada com o nível digital (precisão muito alta) atende as finalidades propostas.

Palavras-chave: rede altimétrica, nivelamento geométrico, precisão.

ABSTRACT - This study aims to evaluate the quality of a geodesic network altimetry within local site to support engineering activities using analog level precision $\pm 2 \mathrm{~mm} / \mathrm{km}$ of double leveling and digital level precision $\pm 0.3 \mathrm{~mm} / \mathrm{km}$ of double leveling, from the analysis of Covariance Matrices residues from adjusted observations and altitudes ajusted. Comparing then following the adjusted heights and unevenness, determined with the analog level to the adjusted heights and unevenness, determined with the digital level. It is noteworthy that this altimetry network is not part of the Brazilian Geodetic System (SGB), composed of twelve level References (RRNN), established and located in the Historical Center of the city of João Pessoa - PB. The bench marks were determined by geometric leveling method and adjusted by the Method of Least Squares, using the model of correlates. With regard to the uncertainties obtained for the altimetric coordinates of reference points implemented by the geometric leveling method with the analog level, they are better than $\pm 0.001 \mathrm{~m}$. Thus, the altimetric network implemented with analog level (high precision) and compared with altimetric network implemented with digital level (very high precision) fulfills the aims proposed.

Keywords: altimetry network, leveling, precision.

\section{INTRODUÇÃO}

A integração de redes altimétricas locais a uma rede altimétrica consistente com os Sistemas Globais de
Altitudes modernos ainda é um problema no Brasil, porque o conceito de altitude está relacionado com o problema de valor de Contorno da Geodésia Física. Esta 
questão precisa ser discutida do ponto de vista geométrico e físico (DE FREITAS; BLITZKOW, 1999).

Em Ferreira (2011) é visto que as altitudes do Sistema Geodésico Brasileiro (SGB) foram obtidas por nivelamento geométrico dissociado de observações gravimétricas. Isto significa que somente a redução ortométrica-normal é aplicada. Um ajustamento rigoroso para as redes de nivelamento requer a transformação para que os desníveis observados resultem em diferenças de potencial usando gravidade ao longo das linhas de nivelamento. Segundo o autor, negligências das reduções do efeito da gravidade para os circuitos de nivelamento resultam em grandes erros nas altitudes pertencentes à RAAP (Rede Altimétrica de Alta Precisão) do IBGE(Instituto Brasileiro de Geografia e Estatística).

O Brasil vem desenvolvendo esforços com o intuito de modernizar seu Sistema de Referência Altimétrico, revisando a definição e realização do mesmo, em parcerias do IBGE e com instituições de ensino e pesquisa. Entretanto um problema a ser resolvido no Brasil é a não uniformidade na distribuição das Referências de Nível da RAAP. Disso, resultam os vazios altimétricos em várias regiões do país, como exemplo, nas regiões Norte, Nordeste e Centro Oeste, onde são encontradas as maiores inconsistências no modelo do geopotencial MAPGEO2010 do Brasil. Essas inconsistências podem estar diretamente ligadas à realização das linhas de nivelamento geométrico nestas regiões (MATOS et al., 2012).

Neste contexto, pesquisadores do Laboratório de Topografia do Instituto Federal de Educação, Ciência e Tecnologia da Paraíba, em parceria com pesquisadores do Laboratório de Geodésia da Universidade Federal de Pernambuco estão implantando e densificando novos circuitos de nivelamento geométrico, no sentido de reduzir o vazio altimétrico existente em João Pessoa - PB e Recife - PE. Com respeito à área deste trabalho, a cidade de João Pessoa, ressalta-se que esta, atualmente, está contemplada com apenas três Referências de Nível pertencentes ao SGB.

A implantação de uma rede altimétrica é importante para melhorar a acurácia dos trabalhos de levantamentos, de forma a garantir a integridade e confiabilidade dos mesmos. A realização deste trabalho justifica-se pela necessidade de se ter coordenadas altimétricas referenciadas ao Sistema de Referência Vertical para a realização de trabalhos de Engenharia na cidade de João Pessoa.

Na temática apresentada o objetivo deste trabalho consiste em analisar a qualidade de uma rede altimétrica, cujos desníveis foram obtidos com nível analógico de precisão $\pm 2 \mathrm{~mm} / \mathrm{km}$ de duplo nivelamento, localizada no Centro Histórico da Cidade de João Pessoa e posteriormente, comparar com as altitudes obtidas com nível digital de precisão $\pm 0,3 \mathrm{~mm} / \mathrm{km}$ de duplo nivelamento, estas últimas determinadas em campanhas anteriores e discutidas em Gama et al. (2013) e Gama et al. (2015).

\section{CONTROLE DE QUALIDADE DA REDE ALTIMÉTRICA}

O controle de qualidade na realização do nivelamento geométrico neste trabalho foi, realizado como descrito em Seixas et al.,(2014) a partir da verificação do nível de luneta empregado. Em loco em função das observações realizadas em campo e seus respectivos desvios; a partir do cálculo do erro de fechamento nos circuitos de nivelamento geométrico e respectivos perímetros realizados e a partir do emprego de visadas equidistantes.

Ao realizar um nivelamento geométrico ao longo de um circuito fechado, a soma dos desníveis deveria ser teoricamente nula. Os erros de medição proporcionam erros de fechamento, que servem como indicador preliminar da precisão das medidas e devem ser distribuídos no ajustamento (GEMAEL,1994; NIEMEIER, 2002).

\subsection{Método dos mínimos quadrados - modelo dos correlatos}

O controle de qualidade da rede altimétrica, neste trabalho, foi realizado aplicando-se as equações do Método dos Mínimos Quadrados - Modelo dos Correlatos. O Modelo dos Correlatos, também denominado Modelo de Equações de Condição, é o modelo utilizado no ajustamento quando se tratam de observações, sujeitas a equações de condição. O modelo matemático que caracteriza as observações condicionadas é descrito por (GEMAEL, 1994) como mostra a Equação1:

$$
\mathrm{F}\left(\mathrm{L}_{\mathrm{a}}\right)=0
$$

A Equação 1 compreende o conjunto de equações de condição a serem satisfeitas pelas observações ajustadas. O Modelo dos Correlatos abrange apenas valores observados ajustados no qual $\mathrm{F}$ representa $r$ funções e o vetor $\mathrm{L}_{\mathrm{a}}$ tem dimensão $n x l$.

Envolvendo os valores observados no modelo, Equação1, obtêm-se as equações de condição transformadas, acrescidas dos resíduos $\mathrm{V}_{\mathrm{i}}$, dada pela Equação 2:

$$
\mathrm{L}_{\mathrm{a}}=L_{b}+V
$$

Pode-se assim, representar o modelo matemático conforme a Equação 3:

$$
\mathrm{F}\left(L_{b}+V\right)=0
$$

A função $\mathrm{F}\left(\mathrm{L}_{\mathrm{b}}\right)$, dos valores observados, representa um erro de fechamento calculado pela Equação 4: 


$$
\mathrm{W}=\mathrm{F}\left(\mathrm{L}_{\mathrm{b}}\right)
$$

Denomina-se por B, a matriz das derivadas parciais, dada pela Equação 5.

$$
B=\frac{\partial F}{\partial L_{a}}(5)
$$

Que corresponde ao modelo linearizado dos correlatos, envolvendo $r$ equações de condições transformadas com $n$ incógnitas, tendo-se as seguintes dimensões apresentadas na Equação 6.

$$
{ }_{r} B_{n} \times{ }_{n} V_{1}+{ }_{r} W_{1}={ }_{r} 0_{1}
$$

Os valores observados ajustados são obtidos a partir do vetor dos resíduos (Equação7), resultando assim, no vetor $L_{a}$ (Equação 2).

$$
V=P^{-1} \cdot B^{T} \cdot K
$$

Sendo:

$P$ a matriz dos pesos;

$K$ são multiplicadores de Lagrange (correlatos).

\subsubsection{Análise da qualidade do ajustamento}

De acordo com Gemael (1994) no ajustamento das observações pelo Método dos Mínimos Quadrados (MMQ), os erros grosseiros e sistemáticos devem ser eliminados das observações antes da realização do ajustamento. Entretanto, para verificar se os referidos erros foram eliminados é necessária à verificação da qualidade dos valores ajustados, utilizando-se procedimentos estatísticos.

A Análise da Qualidade do Ajustamento é baseada na comparação entre $\widehat{\sigma}_{0}^{2}$ (variância da unidade de peso a posteriori) e $\sigma_{0}{ }^{2}$ (variância da unidade de peso a priori). Para esta análise é utilizado o teste Qui-Quadrado $\left(\chi^{2}\right)$ conhecido também como Teste Global do Modelo (TGM) ou Teste n-dimensional (MIKHAIL; GRACIE, 1981; FRASER, 2003).

O teste leva a formação de duas hipóteses, Equação8:

$$
\left\{\begin{array}{l}
H_{0}: E\left\{\hat{\sigma}_{0}^{2}\right\}=\sigma_{0}{ }^{2} \\
H_{1}: E\left\{\hat{\sigma}_{0}^{2}\right\}>\sigma_{0}{ }^{2}
\end{array}\right.
$$

Para a validação de uma das hipóteses, compara-se o valor calculado dado pela Equação 9:

$$
T=\chi_{c}^{2}=\frac{\hat{\sigma}_{0}^{2}}{\sigma_{0}^{2}} g l
$$

Sendo,

gl - o grau de liberdade;

$T=\chi_{c}^{2}$ - Qui-Quadrado calculado.

$\mathrm{Na}$ qual $T$ (Qui-Quadrado calculado) tem distribuição $\chi^{2}$, com graus de liberdade igual a $n$ - $u$, com os valores teóricos da distribuição $\chi_{(S, 1-\alpha)}^{2}$. A hipótese nula $H_{0}$ não é rejeitada, ao nível de significância $\alpha$, no teste estatístico, se: $T<\chi_{(S ; 1-\alpha)}^{2}$. Caso contrário, $T>\chi_{(S ; 1-\alpha)}^{2}, \quad$ significa que há problemas no ajustamento, ou seja, a hipótese alternativa é rejeitada ao nível de significância de $\alpha$. Desta forma, o ajustamento não é aceito, pois existem erros envolvidos.

Caso a hipótese básica seja rejeitada, uma análise criteriosa deve ser realizada para a investigação dos fatores que podem ser a causa da rejeição (GEMAEL, 1994). Uma vez validado o ajustamento são calculadas as matrizes: Covariância das observações dos resíduos $\left(\sum \mathrm{V}\right)$, Covariância dos valores observados ajustados ( $\sum \mathrm{La}$ ), Covariância dos parâmetros ajustados ( $\left.\sum \mathrm{Xa}\right)$.

\section{REALIZAÇÃO DA REDE ALTIMÉTRICA LOCAL}

A metodologia deste trabalho contempla a implantação, medição, ajustamento e análise dos resultados das altitudes ajustadas das Referências de Nível implantadas.

\section{1 Área de estudo}

A justificativa para adoção do Centro Histórico de João Pessoa como área de estudo é a necessidade da densificação de pontos geodésicos de referência, com coordenadas altimétricas conhecidas para o apoio às Obras de Engenharia, aos Levantamentos topográficos/geodésicos e para referenciar os Levantamentos Cadastrais de representação tridimensional das edificações prediais históricas nesta área. A Fig. 1 mostra a localização da área de estudo e a distribuição das Referências de Nível. 


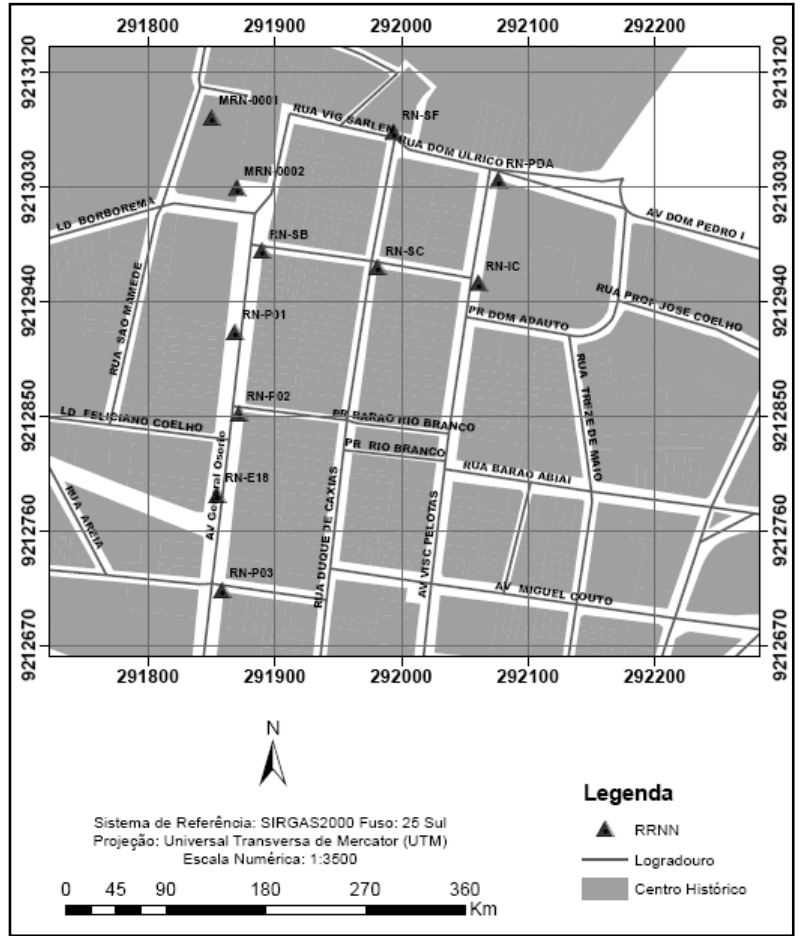

Figura1 - Área de estudo, Centro Histórico de João Pessoa - PB. Fonte: Gama et al. (2014).

\subsection{Medição e processamento da rede altimétrica}

Para a medição da rede de nivelamento foi utilizado o nível analógico TOPCON AT - B4 (precisão $\pm 2 \mathrm{~mm} / \mathrm{km}$ de duplo nivelamento)classificado pela NBR 13133 (ABNT, 1994) NBR 13133 (ABNT, 1994) como nível de precisão alta, mira de alumínio de $4 \mathrm{~m}$ de encaixe e sapatas. Para o ajustamento das observações foi utilizado o software Scilab 5.4.1.

A rede altimétrica é composta por 12 Referências de Nível (Fig. 2) denominadas: RN-396A, MRN0002, MRN0001, RN-SB, RN-E18, RN-SC, RN-IC, RN-PDA, RN-SF, RN-P01, RN-P02 e RN-P03. Sendo a Referência de Nível RN-396A pertencente ao circuito de nivelamento da Rede Altimétrica de Alta Precisão (RAAP) do IBGE, com altitude de 47,2198m $\pm 0,09 \mathrm{~m}$, com data de cálculo em 15/06/2011.

Para a implantação das Referências de Nível foram utilizados pinos de superfícies semi-esféricas, fixadas no meio fio e envolvidas com adesivos epóxis de alta resistência. Os experimentos foram realizados no entorno de 7 quadras urbanas no Centro Histórico de João Pessoa (Fig. 1).

A rede altimétrica, composta por 12 (doze) Referências de Nível, foi mensurada pelo método de nivelamento geométrico. Destas, (9) nove foram medidas com o nível digital Leica DNA - 03 (precisão $\pm 0,3 \mathrm{~mm} / \mathrm{km}$ de duplo nivelamento) classificado pela NBR 13133 (ANBT, 1994) como nível de precisão muito alta e 12 Referências de Nível foram medidas com o nível analógico AT-B4 da TOPCON.

As medições do nivelamento geométrico com o nível digital ocorreram durante a realização dos projetos: Expansão da Estrutura Geodésica Fundamental na Cidade de João Pessoa-PB, Edital n ${ }^{\circ}$ 13/2012 - Pró-Reitora de Pesquisa e Inovação do Instituto Federal de Educação, Ciência e Tecnologia da Paraíba (GAMA, 2014) e A Contribuição da Geodésia Aplicada para a Discretização de Edificações Prediais, edital CNPq/VALE S.A Forma Engenharia $n^{\circ}$ 05/2012, processo $n^{\circ} 454844 / 2012-3$ (SEIXAS, 2014).

O nivelamento com nível digital foi realizado nos dias 30/05/2013 e 31/05/2013, implantando-se uma rede altimétrica com caminhamento do nivelamento ilustrado na Fig. 2.

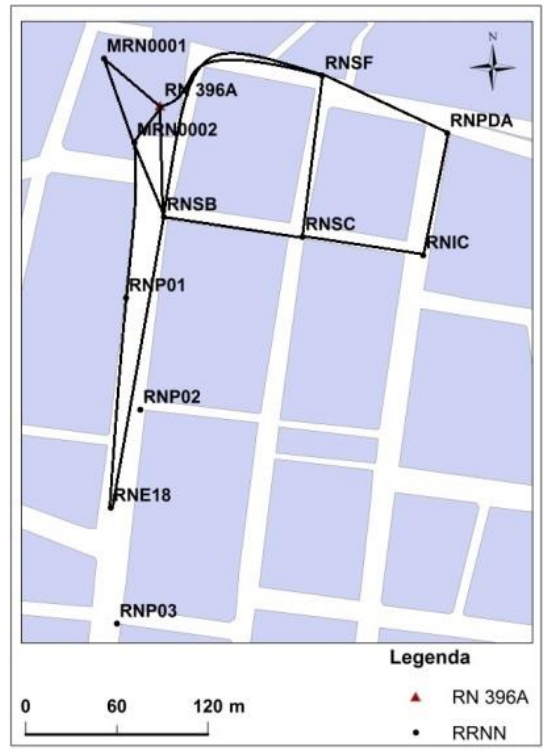

Figura 2 - Caminhamento da rede de nivelamento geométrico com o nível analógico. 
A rede de nivelamento (Figura 2) está composta por dois marcos de concreto, MRN0001 e MRN0002, localizados na Praça da igreja Nossa Senhora das Neves e 6 (seis) pinos metálicos de superfícies semi-esféricas, RNSB, localizado próximo à igreja São Bento, RN-E18, localizado próximo ao Edifício 18, RN-SC, localizado entre as igrejas São Bento e Nossa Senhora do Carmo, RN-IC e RN-PDA, localizados na Praça da igreja Nossa Senhora do Carmo e RN-SF, localizado na calçada da Praça da igreja São Francisco (GAMA et al., 2015). Este nivelamento foi executado partindo do referencial altimétrico do IBGE (RN396A), perfazendo no total 6 (seis) circuitos, compostos por 14 (quatorze) linhas de nivelamento geométrico.

Os circuitos do nivelamento geométrico com o nível digital estão representados na Fig. 3.

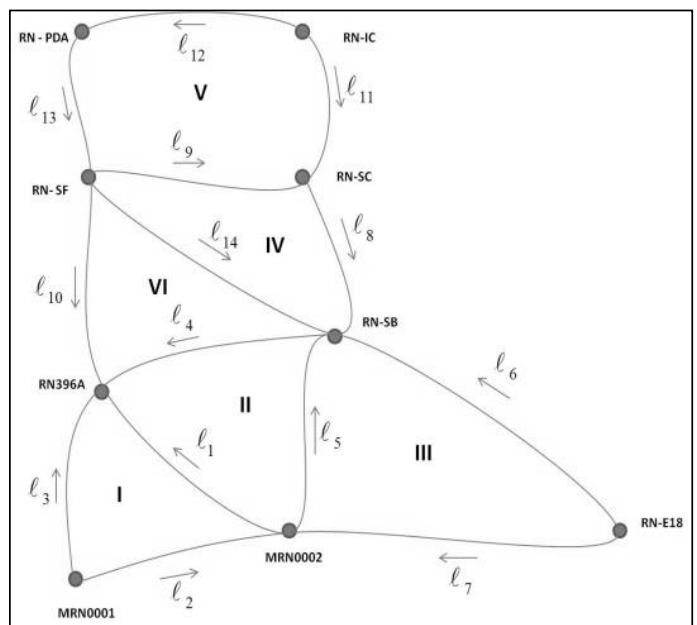

Figura 3 - Circuitos do nivelamento geométrico com o nível digital.

A Tabela 1 apresenta o erro de fechamento e o perímetro para cada circuito independente formado. Todos os erros de fechamento foram abaixo da casa do milímetro (mm).

Tabela 1 - Circuitos do nivelamento, observações, perímetro e seus respectivos erros de fechamento para cada circuito.

\begin{tabular}{cccc} 
Circuito & Linhas & $\begin{array}{c}\text { Perímetro } \\
(\mathrm{Km})\end{array}$ & $\begin{array}{c}\text { Erro de } \\
\text { Fechamento }(\mathrm{m})\end{array}$ \\
\hline I & $l_{1}, l_{2}$ e $l_{3}$ & 0,36588 & 0,00038 \\
II & $l_{1}, l_{4}$ e $l_{5}$ & 0,03474 & 0,00034 \\
III & $l_{5}, l_{6}$, e $l_{7}$ & 0,50549 & $-0,00035$ \\
IV & $l_{9}, l_{8}$ e $l_{14}$ & 0,40840 & 0,00034 \\
V & $l_{9}, l_{11}, l_{12}$ e $l_{13}$ & 0,39494 & 0,00073 \\
VI & $l_{4}, l_{10}$ e $l_{14}$ & 0,37505 & 0,00075 \\
\hline
\end{tabular}

Fonte: (GAMA et al., 2015).

A Tabela 2 mostra as coordenadas altimétrica ajustadas das Referências de Nível desta rede.
Tabela 2 - Altitudes ajustadas e suas incertezas obtidas com nível digital (nível de confiança de 99,5\%)

$\begin{array}{ccc}\begin{array}{c}\text { Referência de } \\ \text { Nível }\end{array} & \mathrm{H}^{\mathrm{a}}(\mathrm{m}) & \text { Incertezas }(\mathrm{m}) \\ \text { MRN0002(H }) & 46,82840 & \pm 0,0001 \\ \text { MRN0001 }\left(H_{C}\right) & 42,69633 & \pm 0,0001 \\ \mathrm{RN}-\mathrm{SB}\left(H_{D}\right) & 47,03758 & \pm 0,0001 \\ \mathrm{RN}-\mathrm{E} 18\left(H_{E}\right) & 45,56069 & \pm 0,0003 \\ \mathrm{RN}-\mathrm{SC}\left(H_{F}\right), & 46,43514 & \pm 0,0002 \\ \mathrm{RN}-\mathrm{SF}\left(H_{G}\right) & 46,28558 & \pm 0,0002 \\ \mathrm{RN}-\mathrm{IC}\left(H_{H}\right) & 44,34809 & \pm 0,0003 \\ \mathrm{RN}-\mathrm{PDA}\left(H_{I}\right) & 45,62494 & \pm 0,0003\end{array}$

Fonte: (GAMA et al., 2015).

O nivelamento geométrico com nível analógico envolve 12 (doze) Referências de Nível (RN-396A, MRN0002, MRN0001, RN-SB, RN-E18, RN-SC, RN-IC, RN-PDA, RN-SF, RN-P01, RN-P02 e RN-P03) formando 9 (nove) circuitos independentes, composto por 20 (vinte) desníveis $\left(\ell_{1}, \ell_{2}, \ell_{3} \ldots \ell_{20}\right)$, também chamadas observações. As referências de Nível RN-P01, RN-P02 e RN-P03estão localizadas na Avenida General Ozório.

$\mathrm{Na}$ Fig. 4 visualiza-se o caminhamento do nivelamento geométrico realizado com o nível analógico.

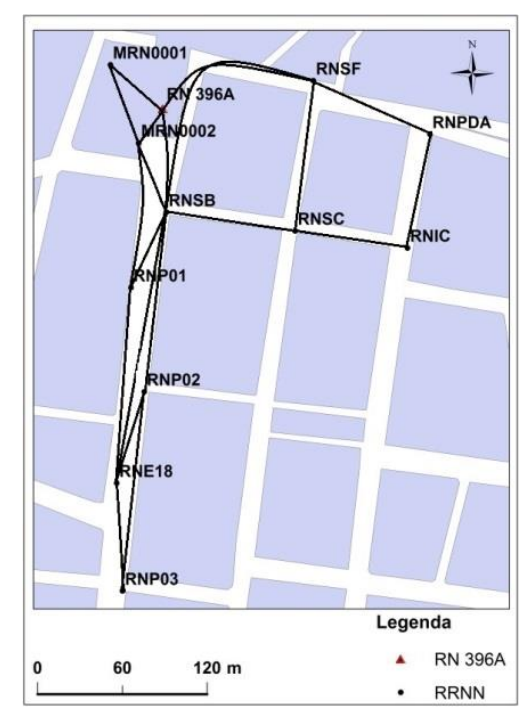

Figura 4 - Caminhamento da rede de nivelamento geométrico com o nível analógico.

As medições com o nível analógico foram realizadas nos dias 30/05/2013 e 31/05/2013. Na Fig. 5 visualizamse os circuitos realizados da rede altimétrica a partir do método de nivelamento geométrico realizados com o nível analógico. 


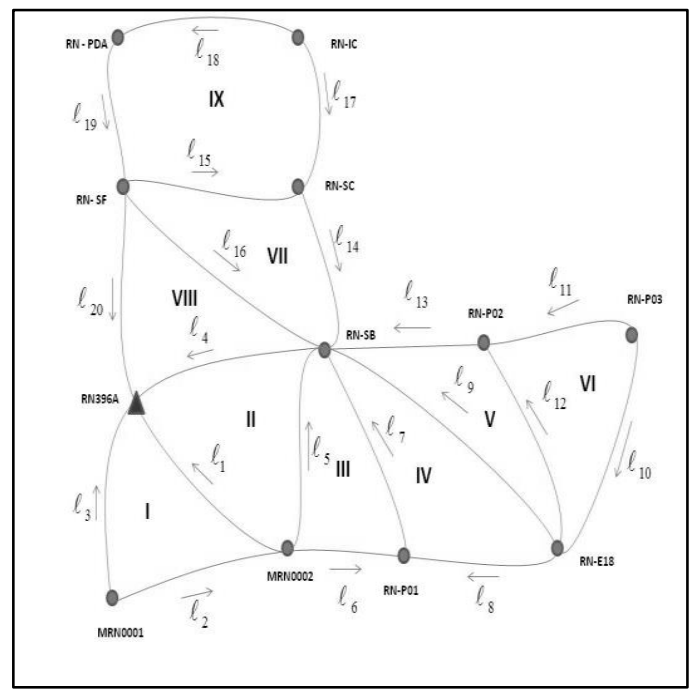

Figura5 - Rede altimétrica definida pelo método do nivelamento geométrico e medida com nível analógico. Fonte: (GAMA et al., 2014).

Para o controle das leituras realizadas nas miras com o nível analógico foi calculada a média entre as leituras dos fios estadimétricos superior e inferior, comparando-a em seguida com a leitura do fio estadimétrico médio lido, sendo tolerável uma diferença menor ou igual a $\pm 0,002 \mathrm{~m}$ entre a média das leituras dos fios superior e inferior calculada e a leitura do fio médio medida. Esta tolerância admitida está de acordo com o critério de estimação das leituras efetuadas na leitura dos fios estadimétricos na mira.

A Tabela 3 apresenta o erro de fechamento e o perímetro para cada circuito independente formado. Todos os erros de fechamento foram menores ou iguais a $\pm 0,002 \mathrm{~m}$.

Tabela 3 - Circuitos do nivelamento, observações, perímetro e seus respectivos erros de fechamento para cada circuito.

\begin{tabular}{cccc}
\hline Circuito & Linhas & $\begin{array}{c}\text { Perímetro } \\
(\mathrm{Km})\end{array}$ & $\begin{array}{c}\text { Erro de } \\
\text { fechamento } \\
(\mathrm{m})\end{array}$ \\
\hline I & $l_{1}, l_{2}, l_{3}$ & 0,1449 & 0,002 \\
II & $l_{1}, l_{4}, l_{5}$ & 0,1591 & 0,000 \\
III & $l_{5}, l_{6}, l_{7}$ & 0,2432 & $-0,001$ \\
IV & $l_{7}, l_{8}, l_{9}$ & 0,4058 & $-0,001$ \\
V & $l_{9}, l_{12}, l_{13}$ & 0,4037 & $-0,001$ \\
VI & $l_{10}, l_{11}, l_{12}$ & 0,2906 & 0,002 \\
VII & $l_{14}, l_{15}, l_{16}$ & 0,4198 & 0,000 \\
VIII & $l_{9}, l_{16}, l_{20}$ & 0,5635 & 0,000 \\
IX & $l_{13}, l_{17}, l_{18}$ e $l_{19}$ & 0,3994 & 0,001 \\
\hline
\end{tabular}

Para o ajustamento da rede de nivelamento utilizouse como observações as diferenças de nível $(\Delta \mathrm{H})$ apresentadas na Tabela 4 .
Tabela 4 - Diferenças de nível entre as RRNN

\begin{tabular}{|c|c|c|c|c|}
\hline Saída & Chegada & $\begin{array}{c}\text { Linha } \\
\text { nivelada }\end{array}$ & $\begin{array}{l}\Delta \mathrm{H} \\
(\mathrm{m})\end{array}$ & Distância $(\mathrm{Km})$ \\
\hline RN-396A & MRN0002 & $l_{1}$ & 0,391 & 0,023 \\
\hline MRN0002 & MRN0001 & $l_{2}$ & 4,120 & 0,060 \\
\hline RN-396A & MRN0001 & $l_{3}$ & 4,513 & 0,061 \\
\hline RN-396A & RN-SB & $l_{4}$ & 0,182 & 0,080 \\
\hline $\mathrm{RN}-\mathrm{SB}$ & MRN0002 & $l_{5}$ & 0,209 & 0,055 \\
\hline RN-P01 & MRN0002 & $l_{6}$ & 0,022 & 0,114 \\
\hline RN-SB & RN-P01 & $l_{7}$ & 0,186 & 0,074 \\
\hline RN-P01 & RN-E18 & $l_{8}$ & 1,291 & 0,129 \\
\hline RN-SB & RN-E18 & $l_{9}$ & 1,476 & 0,202 \\
\hline RN-E18 & RN-P03 & $l_{10}$ & 2,376 & 0,078 \\
\hline RN-P02 & RN-P03 & $l_{11}$ & 3,507 & 0,139 \\
\hline RN-P02 & RN-E18 & $l_{12}$ & 1,129 & 0,073 \\
\hline RN-SB & RN-P02 & $l_{13}$ & 0,346 & 0,129 \\
\hline RN-SB & RN-SC & $l_{14}$ & 0,604 & 0,092 \\
\hline RN-SC & $\mathrm{RN}-\mathrm{SF}$ & $l_{15}$ & 0,149 & 0,126 \\
\hline RN-SB & $\mathrm{RN}-\mathrm{SF}$ & $l_{16}$ & 0,753 & 0,202 \\
\hline RN-SC & RN-IC & $l_{17}$ & 2,086 & 0,092 \\
\hline RN-PDA & RN-IC & $l_{18}$ & 1,277 & 0,082 \\
\hline RN-SF & RN-PDA & $l_{19}$ & 0,661 & 0,097 \\
\hline $\mathrm{RN}-396 \mathrm{~A}$ & $\mathrm{RN}-\mathrm{SF}$ & $l_{20}$ & 0,935 & 0,160 \\
\hline
\end{tabular}

As coordenadas altimétricas da rede de nivelamento foram ajustados pelo Método dos Mínimos Quadrados com emprego das equações de condição, ao nível de $95 \%$ de confiança, seguindo - se com a análise da qualidade com o Teste Global do Modelo (TGM). 
Foram realizadas 20 linhas de nivelamento geométrico, representando os desníveis medidos, considerados neste modelo de ajustamento como 20 observações $\left(\ell_{i}\right)$. As linhas de nivelamento geométrico envolveram 12 (doze) RRNN, sendo a altitude da RN396A considerada fixa, com uma altitude de 47,2198 $\mathrm{m}$, e as demais consideradas incógnitas. Resultam assim, as equações de condição. Sendo $n=20$ (vinte) o número de observações e $u=11$ (onze) o número de incógnitas, resultando o grau de liberdade ( $\mathrm{gl}$ ) igual a 9 (nove). A precisão da Referência de Nível RN396A não foi considerada no ajustamento, neste trabalho.

As equações de condição utilizam os valores das diferenças de nível $(\Delta \mathrm{H})$ entre as RRNN, apresentadas na Tabela4. As equações de condição transformadas adicionadas aos resíduos são escritas de acordo com a Equação 2, resultando nas Equações 10 do modelo matemático:

$$
\left.\begin{array}{l}
l_{1}+l_{2}-l_{3}+v_{1}+v_{2}-v_{3}=0 \\
-l_{1}+l_{4}+l_{5}-v_{1}+v_{4}+v_{5}=0 \\
-l_{5}+l_{6}+l_{7}-v_{5}+v_{6}+v_{7}=0 \\
-l_{7}-l_{8}+l_{9}-v_{7}-v_{8}+v_{9}=0 \\
-l_{9}+l_{12}+l_{13}-v_{9}+v_{12}+v_{13}=0 \\
-l_{10}+l_{11}-l_{12}-v_{10}+v_{11}-v_{12}=0 \\
-l_{14}-l_{15}+l_{16}-v_{14}-v_{15}+v_{16}=0 \\
-l_{4}-l_{16}+l_{20}-v_{4}-v_{16}-v_{20}=0 \\
l_{15}-l_{17}+l_{18}+l_{19+} v_{15}-v_{17}+v_{18}+v_{19}=0
\end{array}\right\}
$$

\subsection{Análise da qualidade da rede altimétrica}

Definidas as matrizes dos coeficientes (B) e vetor de fechamento (W), obtém-se a matriz dos pesos. A matriz dos pesos traz uma importante influência na qualidade do ajustamento. Neste trabalho considera-se uma tolerância altimétrica de $\sigma_{i}= \pm 2 m m \sqrt{k_{i}}$, de acordo com a precisão do nível analógico AT-B4 empregado.

Tendo-se assim, $\sigma_{0}{ }^{2}=4 \times 10^{-6} \times k_{i}$, sendo ainda este mesmo valor adotado como sigma a priori.

Reescrevendo, obtém-se a matriz dos pesos dada pela Equação 11.

$$
P_{20 \times 20}=k_{i}^{-1}
$$

Onde:

$K_{i}$ é Distância nivelada em km;

$P$ é Matriz dos pesos.

Refazendo a Equação 11, chega-se na Equação 12.

$$
P_{20 X 20}=\sigma_{0}^{2}\left[\begin{array}{cccc}
1 / k_{1} & 0 & \ldots & 0 \\
0 & 1 / k_{2} & \ldots & 0 \\
0 & 0 & \ldots & 0 \\
0 & 0 & \ldots & 1 / k_{n}
\end{array}\right]_{20 \times 20}
$$

Com base nestes experimentos, obteve-se a variância da unidade de peso unitário a posteriori $\left(\hat{\sigma}_{0}^{2}\right)$ aplicandose Equação 13.

$$
\hat{\sigma}_{0}^{2}=\frac{\mathrm{V}^{\mathrm{T}} P V}{g l}
$$

Onde:

V é o vetor dos resíduos;

$P$ é a matriz dos pesos;

$g l$ é o grau de liberdade.

Resultando em:

$$
\hat{\sigma}_{0}^{2}=6,8 \times 10^{-6} \text {. }
$$

Para a validação de uma das hipóteses compara-se o valor do $\chi^{2}$ calculado, com os valores teóricos da distribuição $\chi_{(g l, 1-\alpha)}^{2}$.

Utilizando-se o teste Qui-Quadrado, $\chi^{2}$, apresentado na Equação9, com 9 (nove) graus de liberdade tem-se:

$$
\begin{aligned}
& \chi_{\text {calculado }}^{2}=\frac{6,8 \times 10^{-6}}{4 \times 10^{-6}} \times 9 \\
& \chi_{\text {calculado }}^{2}=15,3
\end{aligned}
$$

Utilizando a estimativa por intervalo resultam $\chi_{(9 ; 0,025)}^{2}=2,700$ e $\chi_{(9 ; 0,975)}^{2}=19,023$. O teste é aceito, pois a hipótese nula $\mathrm{H}_{0}$ não foi rejeitada ao nível de significância de $\alpha=5 \%$ e o valor calculado para a forma quadrática $\mathrm{V}^{\mathrm{T}} P V$ de distribuição Qui-Quadrado: $\chi_{c}^{2}=15,3$ está dentro da região de aceitação.

Sendo assim, não foram detectados erros grosseiros nas observações realizadas.

Com o teste aceito são calculadas as Matrizes Variância - Covariância: dos resíduos, das observações ajustadas e das altitudes.

Os resíduos ( $v i$ ) com suas respectivas incertezas das altitudes ajustadas calculadas pela Equação 14 são dados na Tabela 5 .

$$
\sum_{V}=\hat{\sigma}_{0}^{2}\left(P^{-1} \cdot B^{T} \cdot M^{-1} \cdot B \cdot P^{-1}\right)
$$


Tabela 5 - Resíduos das observações ajustadas e suas respectivas incertezas.

\begin{tabular}{|c|c|c|c|c|c|}
\hline$v i$ & $\begin{array}{c}\text { Valores } \\
\text { dos } v i \\
\text { (m) }\end{array}$ & $\begin{array}{l}\text { Incertezas } \\
\text { (m) }\end{array}$ & $v i$ & $\begin{array}{l}\text { Valores } \\
\text { dos } v i \\
\text { (m) }\end{array}$ & $\begin{array}{l}\text { Incertezas } \\
(\mathrm{m})\end{array}$ \\
\hline$v_{1}$ & 0,0002 & $\pm 0,0002$ & $v_{11}$ & $-0,0008$ & $\pm 0,0007$ \\
\hline$v_{2}$ & 0,0009 & $\pm 0,0004$ & $v_{12}$ & 0,0007 & $\pm 0,0004$ \\
\hline$v_{3}$ & $-0,0009$ & $\pm 0,0004$ & $v_{13}$ & 0,0006 & $\pm 0,0006$ \\
\hline$v_{4}$ & 0,0003 & $\pm 0,0005$ & $v_{14}$ & 0,0000 & $\pm 0,0004$ \\
\hline$v_{5}$ & $-0,0001$ & $\pm 0,0004$ & $v_{15}$ & $-0,0003$ & $\pm 0,0007$ \\
\hline$v_{6}$ & 0,0008 & $\pm 0,0006$ & $v_{16}$ & $-0,0002$ & $\pm 0,0009$ \\
\hline$v_{7}$ & 0,0001 & $\pm 0,0004$ & $v_{17}$ & 0,0002 & $\pm 0,0004$ \\
\hline$v_{8}$ & $-0,0008$ & $\pm 0,0006$ & $v_{18}$ & $-0,0002$ & $\pm 0,0003$ \\
\hline$v_{9}$ & 0,0003 & $\pm 0,0009$ & $v_{19}$ & $-0,0003$ & $\pm 0,0004$ \\
\hline$v_{10}$ & 0,0005 & $\pm 0,0004$ & $v_{20}$ & 0,0001 & $\pm 0,0008$ \\
\hline
\end{tabular}

As observações ajustadas calculadas pela Equação 15 são dadas na Tabela 6.

$$
\Sigma_{L_{a}}=\hat{\sigma}_{0}^{2}\left(P^{-1}-P^{-1} \cdot B^{T} \cdot M^{-1} \cdot B \cdot P^{-1}\right)
$$

Tabela 6 - Desníveis ajustados e respectivas incertezas.

\begin{tabular}{cccccc}
\hline$l_{i}$ & $\Delta \mathrm{H}^{\mathrm{a}}(\mathrm{m})$ & $\begin{array}{c}\text { Incertezas } \\
(\mathrm{m})\end{array}$ & $l i$ & $\Delta \mathrm{H}^{\mathrm{a}}(\mathrm{m})$ & $\begin{array}{c}\text { Incertez } \\
\mathrm{as}(\mathrm{m})\end{array}$ \\
\hline$l_{1}^{a}$ & 0,391 & $\pm 0,0003$ & $l_{11}^{a}$ & 3,506 & $\pm 0,0007$ \\
$l_{2}^{a}$ & 4,121 & $\pm 0,0004$ & $l_{12}^{a}$ & 1,130 & $\pm 0,0005$ \\
$l_{3}^{a}$ & 4,512 & $\pm 0,0004$ & $l_{13}^{a}$ & 0,347 & $\pm 0,0007$ \\
$l_{4}^{a}$ & 0,182 & $\pm 0,0004$ & $l_{14}^{a}$ & 0,604 & $\pm 0,0006$ \\
$l_{5}^{a}$ & 0,209 & $\pm 0,0004$ & $l_{15}^{a}$ & 0,149 & $\pm 0,0006$ \\
$l_{6}^{a}$ & 0,023 & $\pm 0,0005$ & $l_{16}^{a}$ & 0,753 & $\pm 0,0006$ \\
$l_{7}^{a}$ & 0,186 & $\pm 0,0005$ & $l_{17}^{a}$ & 2,086 & $\pm 0,0007$ \\
$l_{8}^{a}$ & 1,290 & $\pm 0,0007$ & $l_{18}^{a}$ & 1,277 & $\pm 0,0006$ \\
$l_{9}^{a}$ & 1,476 & $\pm 0,0006$ & $l_{19}^{a}$ & 0,661 & $\pm 0,0007$ \\
$l_{10}^{a}$ & 2,376 & $\pm 0,0006$ & $l_{20}^{a}$ & 0,935 & $\pm 0,0007$ \\
\hline
\end{tabular}

O maior e o menor resíduo foram encontrados para as observações $l_{2}, L_{3}$ e $l_{14}$ com valores, respectivamente, de $0,0009 \mathrm{~m} ;-0,0009 \mathrm{~m}$ e $0 \mathrm{~m}$.

As altitudes ajustadas das RRNN são obtidas, somando-se os respectivos desníveis ajustados, "independente do caminho percorrido".

$$
\begin{aligned}
& M R N 0002=R N 396 A-\ell^{a}{ }_{1} \\
& M R N 0001=R N 396 A-\ell^{a}{ }_{1}-\ell^{a}{ }_{2} \\
& R N-S B=R N 396 A-\ell^{a}{ }_{4} \\
& R N-P 01=R N 396 A-\ell^{a}{ }_{4}-\ell^{a}{ }_{7} \\
& R N-E 18=R N 396 A-\ell^{a}{ }_{4}-\ell^{a}{ }_{9} \\
& R N-P 03=R N 396 A-\ell^{a}{ }_{4}-\ell^{a}{ }_{9}-\ell^{a}{ }_{10} \\
& R N-P 02=R N 396 A-\ell^{a}{ }_{4}-\ell^{a}{ }_{13} \\
& R N-S C=R N 396 A-\ell^{a}{ }_{4}-\ell^{a}{ }_{14} \\
& R N-I C=R N 396 A-\ell^{a}{ }_{4}-\ell^{a}{ }_{14}-\ell^{a}{ }_{17} \\
& R N-P D A=R N 396 A-\ell^{a}{ }_{4}-\ell^{a}{ }_{14}-\ell^{a}{ }_{17}+\ell^{a}{ }_{18} \\
& R N-S F=R N 396 A-\ell^{a}{ }_{20}
\end{aligned}
$$

A Matriz Variância - Covariância das altitudes ajustadas $\sum H$ é obtida utilizando-se a Equação 16, a partir da propagação dos erros nas equações anteriores.

$$
\sum H=G \cdot \sum L_{a} \cdot G^{\prime}
$$

Sendo,

$\mathrm{G}$ a matriz dos coeficientes;

$\sum L_{a}$ a Matriz Variância - Covariância dos desníveis ajustados.

As altitudes ajustadas e suas respectivas incertezas são apresentadas na Tabela 7 .

Tabela 7 - Altitudes ajustadas e suas incertezas, obtidas com o nível analógico, com nível de confiança de $95 \%$.

\begin{tabular}{ccc}
\hline $\begin{array}{c}\text { Referência de } \\
\text { Nível }\end{array}$ & $\mathrm{H}^{\mathrm{a}}(\mathrm{m})$ & Incertezas $(\mathrm{m})$ \\
\hline MRN0002 & 46,829 & $\pm 0,0003$ \\
MRN0001 & 42,708 & $\pm 0,0004$ \\
RNSB & 47,037 & $\pm 0,0004$ \\
RN-P01 & 46,851 & $\pm 0,0006$ \\
RNE18 & 45,561 & $\pm 0,0008$ \\
RN-P03 & 43,185 & $\pm 0,0009$ \\
RN-P02 & 46,691 & $\pm 0,0008$ \\
RNSC & 46,433 & $\pm 0,0007$ \\
RNIC & 44,347 & $\pm 0,0009$ \\
RNPDA & 45,624 & $\pm 0,0009$ \\
RNSF & 46,285 & $\pm 0,0007$ \\
\hline
\end{tabular}

As incertezas provenientes da Matriz Covariância das altitudes ajustadas (Tabela 6) são menores ou iguais a $0,0009 \mathrm{~m}$.

Os desníveis ajustados e obtidos a partir das medições com o nível digital são mostrados na Tabela 8 .

Tabela 8 - Desníveis ajustados e respectivas incertezas. 


\begin{tabular}{ccl}
\hline $\begin{array}{c}\text { Observações } \\
\text { Ajustadas }\end{array}$ & $\Delta \mathrm{H}^{\mathrm{a}}(\mathrm{m})$ & Incertezas $(\mathrm{m})$ \\
\hline$l_{1}^{a}$ & 0,3914 & $\pm 0,0001$ \\
$l_{2}^{a}$ & 4,1320 & $\pm 0,0001$ \\
$l_{3}^{a}$ & 4,5234 & $\pm 0,0001$ \\
$l_{4}^{a}$ & 0,1822 & $\pm 0,0001$ \\
$l_{5}^{a}$ & 0,2092 & $\pm 0,0001$ \\
$l_{6}^{a}$ & 1,4768 & $\pm 0,0003$ \\
$l_{7}^{a}$ & 1,2677 & $\pm 0,0003$ \\
$l_{8}^{a}$ & 0,6024 & $\pm 0,0002$ \\
$l_{9}^{a}$ & 0,1495 & $\pm 0,0002$ \\
$l_{10}^{a}$ & 0,9342 & $\pm 0,0002$ \\
$l_{11}^{a}$ & 2,0870 & $\pm 0,0002$ \\
$l_{12}^{a}$ & 1,2768 & $\pm 0,0002$ \\
$l_{13}^{a}$ & 0,6606 & $\pm 0,0002$ \\
$l_{14}^{a}$ & 0,7519 & $\pm 0,0002$ \\
\hline
\end{tabular}

Fonte: (GAMA et al., 2015).

Os desníveis ajustados e obtidos a partir das medições com o nível digital (Tabela 8) foram comparados com os desníveis ajustados e obtidos a partir das medições com o nível analógico (Tabela 6), resultando nas discrepâncias mostradas na Tabela 9.

Tabela 9 - Discrepâncias das diferenças de nível entre as RRNN obtidas com nível digital e nível analógico.

\begin{tabular}{cc}
\hline Desníveis Ajustados $\left(\Delta \mathrm{H}^{\mathrm{a}}\right)$ & Discrepâncias $(\mathrm{m})$ \\
\hline RN-396A - MRN0002 & 0,0004 \\
MRN0002 - MRN0001 & 0,0110 \\
RN-396A - MRN0001 & 0,0114 \\
RN-396A - RN-SB & 0,0002 \\
RN-SB - MRN0002 & 0,0002 \\
RN-SB - RN-SC & 0,0016 \\
RN-SC - RN-SF & 0,0005 \\
RN-SB - RN-SF & 0,0002 \\
RN-SC - RN-IC & 0,0010 \\
RN-PDA - RN-IC & 0,0002 \\
RN-SF - RN-PDA & 0,0004 \\
RN-396A - RN-SF & 0,0008 \\
\hline
\end{tabular}

Analisando a Tabela 9, os valores das discrepâncias entre os desníveis determinados com nível digital e com nível analógico variam de 0,001 a $0,0114 \mathrm{~m}$.

As altitudes das Referências de Nível MRN0002, MRN0001, RN-SB, RN-E18, RN-SC, RN-IC, RN-PDA, RNSF, calculadas a partir da realização do nivelamento geométrico com nível digital (Tabela 2) foram comparadas com as altitudes das Referências de Nível determinadas com nível analógico (Tabela 7), obtendo-se as discrepâncias mostradas na Tabela 10.
Tabela 10 - Discrepâncias das altitudes ajustadas das RRNN obtidas com nível digital e nível analógico.

\begin{tabular}{cc}
\hline Referências de Nível & Discrepância $(\mathrm{m})$ \\
\hline MRN0002 & 0,0006 \\
MRN0001 & 0,0117 \\
RN-SB & 0,0006 \\
RN-E18 & 0,0003 \\
RN-SC & 0,0021 \\
RN-IC & 0,0006 \\
RN-PDA & 0,0011 \\
RN-SF & 0,0010 \\
\hline
\end{tabular}

Analisando a Tabela 10, os valores das discrepâncias entre as altitudes determinadas com nível digital e com nível analógico variam de 0,0003 a 0,0114m.

A linha de nivelamento $l_{2}$ realizada com nível analógico apresenta um desnível de 4,120m, enquanto que esta mesma linha medida com nível digital tem desnível de 4,13190m.

Apesar das incertezas das altitudes ajustadas (Tabela 6) serem inferiores a $\pm 0,001 \mathrm{~mm}$, sendo assim condizentes com a incerteza esperada quando se utiliza nível analógico de alta precisão e miras centimetradas, como utilizados neste trabalho para a realização de redes de nivelamento geométrico, a discrepância entre as altitudes ajustadas da Referência de Nível MRN0001 e as discrepâncias entre os desníveis ajustados das linhas de nivelamento geométrico $l 1$ e $l 2$ estão na ordem de $\pm 0,01 \mathrm{~m}$. Uma hipótese para este ocorrido é que a Referência de Nível MRN0001 pode ter sofrido um rebaixamento após a medição realizada com o nível digital, ocasionada pelo tipo de materialização utilizada para a definição da Referência de Nível MRN0001 e solo no seu entorno.

Tomando as discrepâncias da Tabela 7 pode-se dizer que o nível analógico de alta precisão pode ser empregado para a determinação de altitudes para apoio as atividades de Engenharia.

\section{CONCLUSÕES}

O ajustamento das coordenadas altimétricas das Referências de Nível foi realizado pelo Método dos Mínimos Quadrados, utilizando o modelo dos correlatos.

Pode-se afirmar que o nível analógico de alta precisão utilizado neste trabalho, pode ser empregado para trabalhos de Engenharia em que a precisão de \pm $2 \mathrm{mmx}(\mathrm{k})^{0,5}$ é aceitável. Neste trabalho as incertezas das altitudes ajustadas (Tabela 7) foram inferiores a $\pm 0,001 \mathrm{~mm}$, ou seja, $\pm 1 \mathrm{~mm}$. Tornando-se desnecessário o emprego do nível digital de precisão muito alta.

\section{AGRADECIMENTO}

A Pró-Reitoria de Pesquisa e Inovação do Instituto Federal de Educação, Ciência e Tecnologia da Paraíba (IFPB), Edital $n^{\circ} 13 / 2012$, pela concessão de bolsa para a realização do projeto de Pesquisa Expansão da Estrutura Geodésica Fundamental na Cidade de João Pessoa-PB, Edital $n^{\circ}$ 13/2012. E à Coordenação de Transportes pela 
concessão do veículo para a realização dos trabalhos de campo. Ao CNPQ/VALE S.A. pela realização do Projeto de Pesquisa: A Contribuição da Geodésia Aplicada para a Discretização de Edificações Prediais, edital CNPq/VALE S.A Forma - Engenharia $n^{\circ}$ 05/2012, processo $n^{\circ}$ 454844/2012-3, pelo recurso financeiro disponibilizado e pelas bolsas ITI-B e ATP-B.

Aos discentes do Curso Superior de Tecnologia em Geoprocessamento do IFPB, Marcio Petterson Marques Oliveira e Michelle Gomes de Araújo pelo auxílio na realização do nivelamento geométrico com o nível analógico.

\section{REFERÊNCIAS BIBLIOGRÁFICAS}

ABNT: NBR-13133. Normas Técnicas para a Execução de Levantamentos Topográficos. 1994.

FRASER, C. S. Least-Squares \& Network Analysis.Class Notes.University of Melbourne.Australia, 2003.

FERREIRA, V.G. Solução do Tipo Brovar para o Segundo Problema de Valor de Contorno da Geodésia com Vistas à Modernização de Sistemas de Altitudes. Tese de Doutorado apresentado ao Programa de Pós Graduação em Ciências Geodésicas. UFPR. 2011.

FREITAS, S.R.C.; BLITZKOW, D. Altitudes e geopotencial. IGeS Bulletin - Special Issue for South America, n. 9, 1999, p. 47-62.

GAMA, L. F.; SEIXAS, A.; SILVA, E. V.; MARQUES, M. P.; ARAUJO, M. G. Implantação e Análise de Estruturas Geodésicas Altimétricas Medidas com Nível Digital. in: VIII colóquio brasileiro deciências geodésicas, 2013, Curitiba - PR.

GAMA, L. F. Relatório Técnico Final: Expansão da Estrutura Geodésica Fundamental na Cidade de João Pessoa-PB, Edital n ${ }^{\circ}$ 13/2012 - Pró-Reitora de Pesquisa e Inovação do Instituto Federal de Educação, Ciência e Tecnologia da Paraíba, 2014.

GAMA, L.F.; SEIXAS, A.; MORAES, J.N.; SILVA, E.V.; GALVÃO, T. B. Controle de Qualidade de Levantamentos Cadastrais Para a Homogeneização de Parcelas Territoriais Urbanas. Revista Brasileira de Cartografia, No. 67/2, p. 287-305, abril/maio. 2015.

GEMAEL, C. Introdução ao ajustamento de observações: aplicações geodésicas. Curitiba: Editora da UFPR, 1994. 319p.

MATOS, A. C. O. C.; BLITZKOW, D.; GUIMARÃES, G. N.; LOBIANCO, M. C. B.; COSTA, S. M. C. Validação do MAPGEO2010 e Comparação com Modelos do Geopotencial Recentes. Bol. Ciênc. Geod., sec. Artigos, Curitiba, v. 18, n¹, p.101-122, jan-mar, 2012.

MIKHAIL, E. M.; GRACIE, G. Analysis and adjustment of survey measurements.New York: Van Nostrand Reinhold Company, 1981.

NIEMEIER, W. Ausgleichungsrechnung. New York: De GruyterLehrbuch. 2002.

SEIXAS,A.; GAMA,L.F.; NAVES, J.M.; SOUZA, A.M. O Estabelecimento de Padrões de Referência Altimétrica Utilizando o Nivelamento Geométrico Para A Definição de Alvos Altos e Inacessíveis. Bol.
Ciênc. Geod., sec. Artigos, Curitiba, v. 20, n² , p.388410, abr-jun, 2014.

SEIXAS, A. de. Relatório Técnico Final: A Contribuição da Geodésia Aplicada para a Discretização de Edificações Prediais. Projeto CNPq/VALE S.A. Forma Engenharia $n^{\circ}$ 05/2012. Processo $n^{\circ}$ 454844/2012-3, 2014. 